\title{
Experiences and opinions of Video Aided Review of Resuscitation Emergencies
}

\author{
Matthew Ibrahim, Resuscitation Lead \\ Phillip Chandler, Resuscitation Practitioner
}

\section{Background}

Learning and debriefing from real life medical emergencies and cardiac arrests are a vital part of advanced resuscitation. It offers valuable insight into technical skills performance of individuals during the resuscitation processes. However, it is more difficult to learn and debrief the non-technical areas. e.g. the communication and interaction between team members during the stressful, high pressure environment of emergencies and resuscitation. This area is often subject to many of the verbal and non-verbal variables which can affect both how a communicator offers their valuable package of instructions and how the receiver interprets that package for use.

Our busy District General Hospital provides its staff with a varied simulation-based learning program, which offered us an opportunity to canvass opinions based on experience. Our staff are integral in developing any new strategies that can impact patient care and therefore we needed to assess their thoughts, feelings and personal experiences of Video Aided Review of Resuscitation Events (VARRE) as our intention is to introduce a body worn camera system to aid this process.

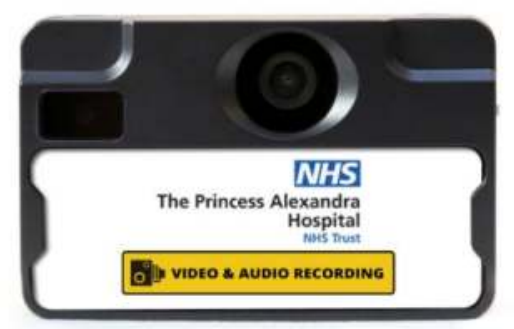

\section{Method}

We surveyed a cross section of our in-hospital staff who may be present at the events using survey monkey ${ }^{\odot}$. The questions used, focused predominantly upon individual's feelings regarding the use of the process and how it could improve patient care, or why it would not.

\section{Results}

There were 77 respondents from a combination of ward areas, ED, critical care/theatres and roaming roles. Over $48 \%$ had been involved in a recorded emergency event, and of those, $90 \%$ had been during simulation. Overall, those that had been involved felt that this process had improved their patient care. Further results are graphed

\section{How would you feel if you knew VARRE was being used in your clinical environment during a clinical emergency?}

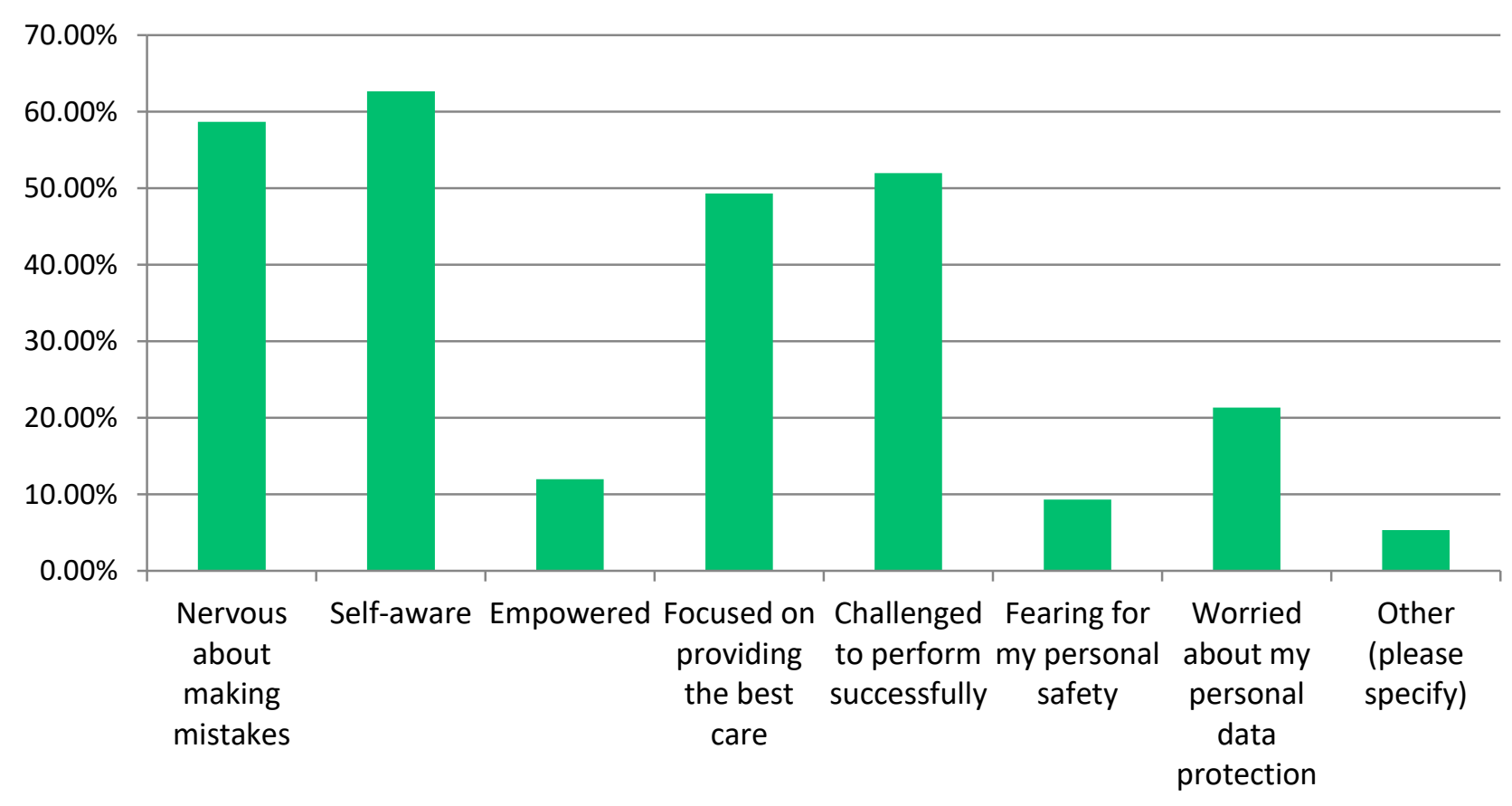

Significantly, of the respondents, nearly $95 \%$ felt that VARRE could benefit patient care. The positive responses far outweighed any concerns expressed.

\section{How do you think it would improve patient care}

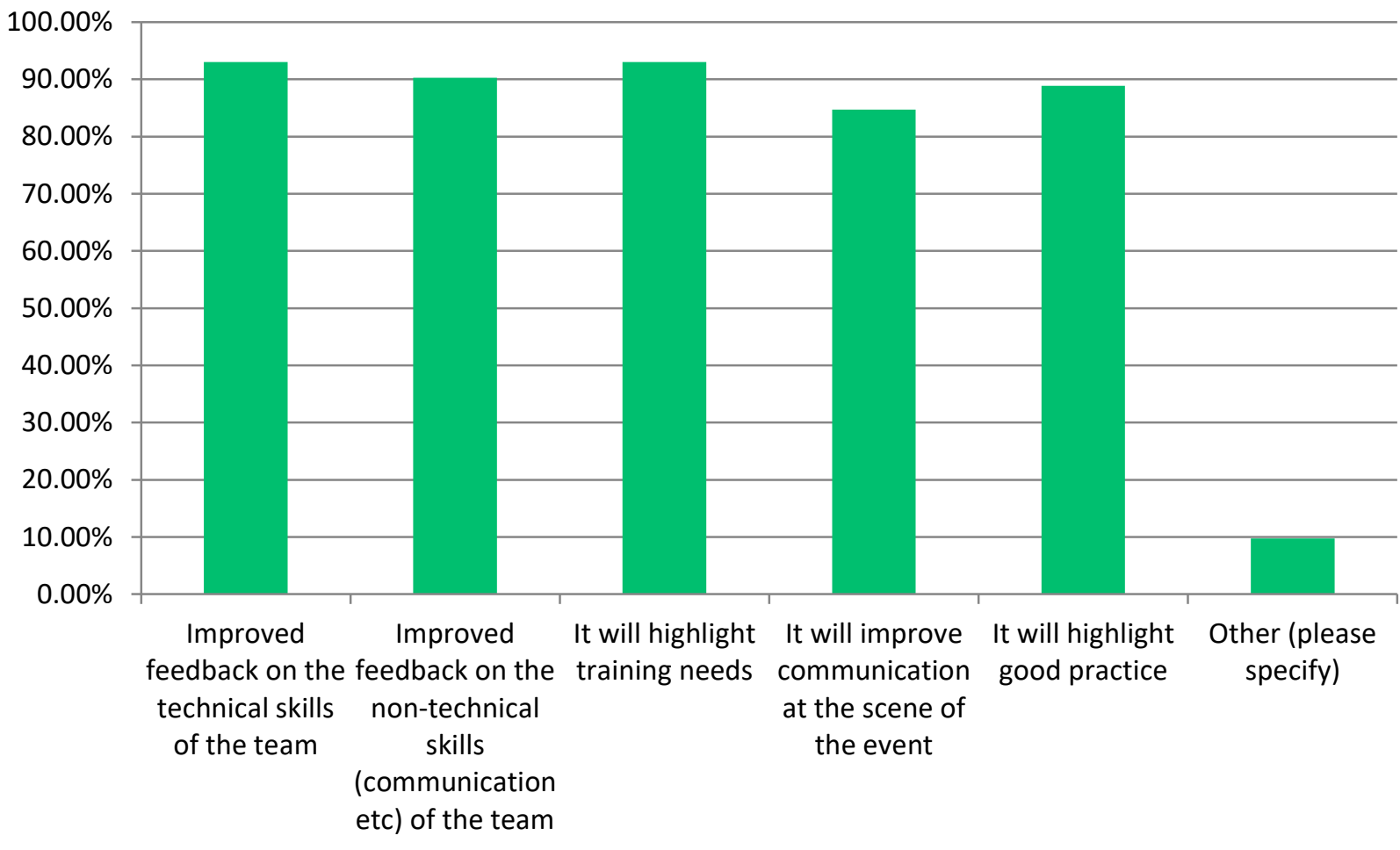

Why do you think it will not improve patient care?

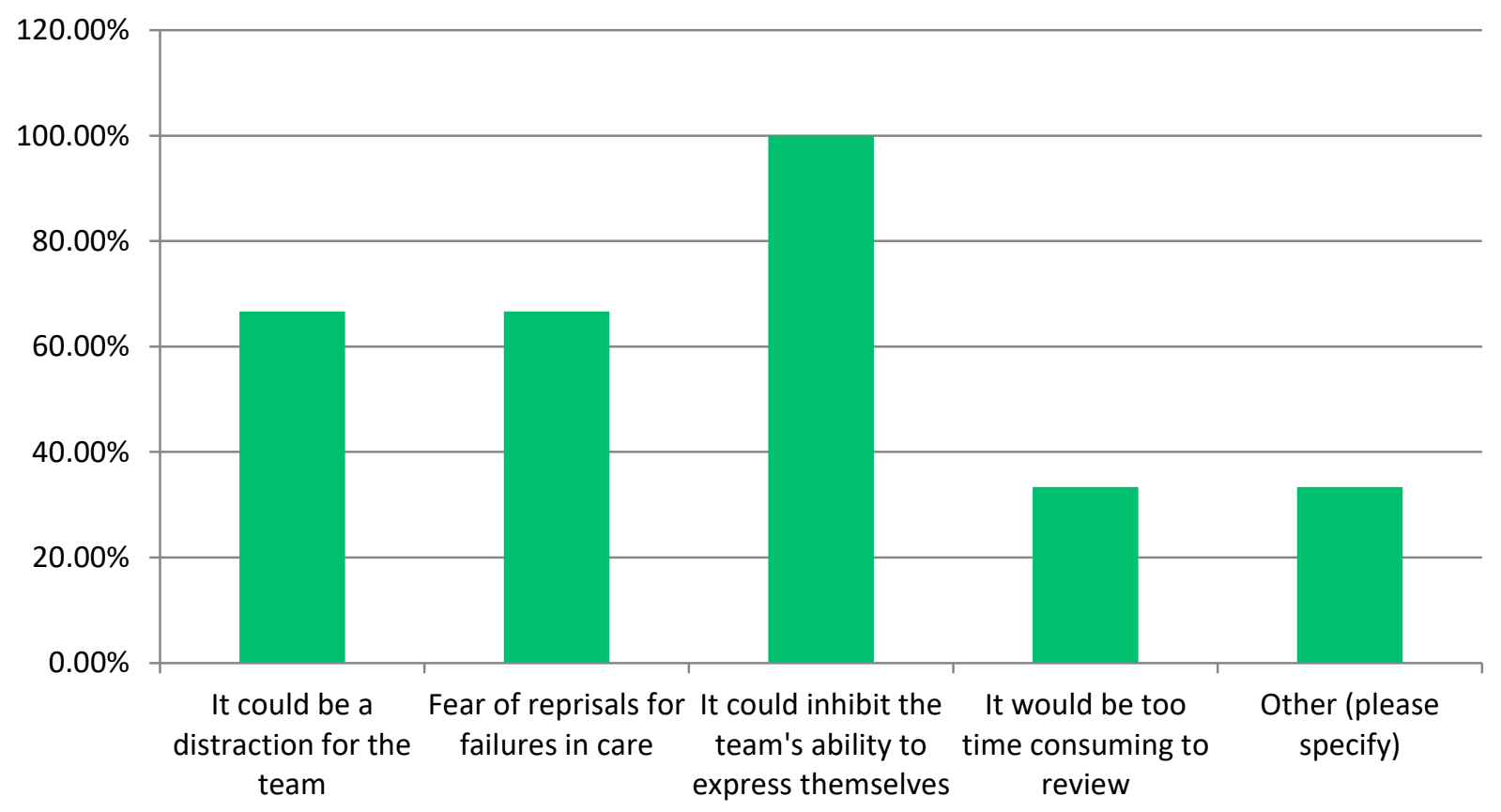

\section{Conclusion}

The positive expressions regarding the use of VARRE were very encouraging and gave us a useful insight into how our colleagues will need to be reassured that the feedback offered is positive, supportive and the use of data is judicious and appropriate.

\section{Discussion}

The introduction and implementation of VARRE poses many challenges. These will range from compliance with Information Governance and Data Protection through to having a tangible review process to reference. But the objective of the process should always remain at the forefront of our minds.

Improved patient care during medical and resuscitation emergencies by supporting our staff to reflect on their own practice and improve in a safe working environment.

Listening to our staff and engaging actively with their concerns and ideas will smooth our road to implementation, and eventually, buy in to the process as a whole. 